\title{
ROUGH ISOMETRY BETWEEN GROMOV HYPERBOLIC SPACES AND UNIFORMIZATION
}

\author{
Jeff Lindquist and Nageswari Shanmugalingam \\ University of Cincinnati, Department of Mathematical Sciences \\ P.O. Box 210025, Cincinnati, OH 45221-0025, U.S.A.; jlindquistmath@gmail.com \\ University of Cincinnati, Department of Mathematical Sciences \\ P.O. Box 210025, Cincinnati, OH 45221-0025, U.S.A.; shanmun@uc.edu
}

\begin{abstract}
In this note we show that given two complete geodesic Gromov hyperbolic spaces that are roughly isometric and an arbitrary $\varepsilon>0$ (not necessarily small), either the uniformization of both spaces with parameter $\varepsilon$ results in uniform domains, or else neither uniformized space is a uniform domain. The terminology of "uniformization" is from [BHK], where it is shown that the uniformization, with parameter $\varepsilon>0$, of a complete geodesic Gromov hyperbolic space results in a uniform domain provided $\varepsilon$ is small enough.
\end{abstract}

\section{Introduction}

Uniform domains play a special role in the study of planar quasiconformal mappings (see for example [MS] where the concept of uniform domains was first introduced, [Mar, GeO, BKR, H, KL]) and in potential theory (see for example [KP, KT, LLMS, A1, A2, HK, BSh]). The notion of uniform domains does not require the underlying space to be Euclidean or smooth, and so has a natural extension to general metric spaces, see Definition 2.4 below. On the other hand, the notion of curvature, as defined in Riemannian geometry, is a second order calculus notion and so does not easily lend itself to the setting of more general metric spaces. Instead, in that non-smooth setting, the role of negative curvature is played by two possible alternatives, Alexandrov curvature and Gromov hyperbolicity, see the discussions in [BH, BuS, CDP, GH]. Gromov hyperbolic spaces were first defined in [Gr] in the context of studying hyperbolic groups.

The work [BHK] demonstrates a strong connection between Gromov hyperbolic spaces and uniform domains. It was shown there that uniform domains in metric spaces, equipped with the quasihyperbolic metric $k$ (see (1)) are necessarily Gromov hyperbolic spaces. Conversely, given a geodesic Gromov hyperbolic space $X$, there is a positive number $\varepsilon_{0}$ such that whenever $0<\varepsilon \leq \varepsilon_{0}$, the uniformization $X_{\varepsilon}$ of $X$ corresponding to the parameter $\varepsilon$ is a uniform domain.

It is not difficult to see that if $X$ and $Y$ are two complete geodesic spaces with $Y$ a Gromov hyperbolic space, and if there is a rough isometry $\Phi: Y \rightarrow X$ as in Definition 2.6, then $X$ is also Gromov hyperbolic; that is, Gromov hyperbolicity is a large scale property and is not destroyed by small-scale perturbations. Therefore it is

https://doi.org/10.5186/aasfm.2021.4624

2020 Mathematics Subject Classification: Primary 53C23; Secondary 30L05.

Key words: Gromov hyperbolic, uniform domain, rough isometry, uniformization.

The second author was partially supported by grant DMS \#1800161 from NSF (U.S.A.) Part of the work for this project was done while the second author was visiting IMPAN; she thanks that institution and the Simons Foundation grant 346300 to IMPAN and the matching 2015-2019 Polish MNiSW fund for their kind hospitality. The authors also thank the referees for suggestions that helped improve the exposition. 
natural to ask whether the allowable range of uniformization parameters is preserved by rough isometries. This is the goal of this current note. In particular, we show that if $X$ and $Y$ are Gromov hyperbolic and $\Phi: Y \rightarrow X$ is a rough isometry, and if $\varepsilon>0$ is such that $X_{\varepsilon}$ is a uniform domain, then $Y_{\varepsilon}$ is also a uniform domain, see Theorem 3.8. In [BBS] it was shown that if a Gromov hyperbolic space $X$ is uniformized to a uniform domain $X_{\varepsilon}$ (for sufficiently small $\varepsilon>0$ ), and the subsequent boundary $Z:=\partial X_{\varepsilon}$ has a hyperbolic filling $Y$ with appropriate scaling parameters, then $Y$ is roughly isometric to $X$. It follows from our results then that $Y_{\varepsilon}$ is also a uniform domain (since we know that $X_{\varepsilon}$ is). It is not difficult to see that $\partial Y_{\varepsilon}$ is isometric to $\partial X_{\varepsilon}$, and hence our result ties the potential theoretic properties of $\partial X_{\varepsilon}$ to those of $Y_{\varepsilon}$, even though $X_{\varepsilon}$ itself could be ill-connected from the point of view of potential theory. It was shown in [BBS] that if $Y_{\varepsilon}$ is a uniform domain, then $Y_{\varepsilon}$ has a suitable measure with respect to which $Y_{\varepsilon}$ is doubling and supports a 1-Poincaré inequality.

If $Z$ is a doubling metric measure space supporting a $p$-Poincaré inequality, then the correct setting for potential theory on $Z$ is the so-called Newton-Sobolev class of functions, see for example [BB]. When $Z$ does not support such a Poincaré inequality, for example if $Z$ does not have sufficiently many rectifiable curves, then the NewtonSobolev class is the wrong class for potential theory on $Z$; in this case, the more appropriate function class is a Besov class of functions on $Z$. See for example [GKS, BBS, BBS2] for more on Besov classes. There are Gromov hyperbolic spaces $X$ for which $X_{\varepsilon}$ is a uniform domain but $\partial X_{\varepsilon}$ may not even be connected; hence the potential theory on $\partial X_{\varepsilon}$ should be via Besov classes. As Besov energies are nonlocal, their properties are not well understood. For example, what metric properties of subsets of $\partial X_{\varepsilon}$ guarantee that the Besov capacity of the set is null? Should $Y$ be another Gromov hyperbolic metric space equipped with a uniformly locally doubling measure supporting a uniformly local Poincaré inequality, and $X$ is roughly isometric to $Y$, then we know from our main theorem, Theorem 3.8, that $Y_{\varepsilon}$ is also a uniform space. It then follows from the results in [BBS2] that the induced measure on $Y_{\varepsilon}$ is doubling and supports a Poincaré inequality; the results of [BBS2] show that the trace of the Newton-Sobolev class of functions on $Y_{\varepsilon}$ is a Besov class on $\partial Y_{\varepsilon}$, and that a subset of $\partial Y_{\varepsilon}$ is null for this Besov class if and only if it is null for the NewtonSobolev class. Null sets for Newton-Sobolev classes are reasonably well understood, and this understanding translates to a reasonable understanding of Besov-null sets in $\partial Y_{\varepsilon}$. Since $X$ is roughly isometric to $Y$, we must have that $\partial X_{\varepsilon}$ is biLipschitz equivalent to $\partial Y_{\varepsilon}$, and so such an understanding can be imported to $\partial X_{\varepsilon}$ as well. See $[\mathrm{BP}]$ for connections between Besov classes on $\partial X_{\varepsilon}$ and the algebraic structure of $X$ for certain geometric classes of hyperbolic spaces $X$.

Observe that by the results of [BHK], $Y_{\varepsilon}$ is a uniform domain if $\varepsilon$ is small enough, but here we do not require smallness of $\varepsilon$. The key reason in [BHK] for requiring $\varepsilon$ be sufficiently small is that for small enough $\varepsilon$ a Gehring-Hayman property holds for hyperbolic geodesics. Since we do not assume $\varepsilon$ to be small, we cannot rely on this property; instead, our proof uses the technique of discretization of paths.

The next section is devoted to providing the relevant definitions. The first part of the third section develops the tools necessary to prove our main theorem, Theorem 3.8, and the proof of that theorem is given in the last part of that section. The last section is devoted to showing that replacing rough isometries with rough quasiisometries makes the conclusion of Theorem 3.8 false. 
We adopt the convention that $Q_{1} \gtrsim Q_{2}$ if there is a constant $C>0$ such that $C Q_{1} \geq Q_{2}$. We say that $Q_{1} \lesssim Q_{2}$ if $Q_{2} \gtrsim Q_{1}$, and we say that $Q_{1} \simeq Q_{2}$ if $Q_{1} \gtrsim Q_{2}$ and $Q_{1} \lesssim Q_{2}$. We say that $Q_{1} \simeq Q_{2}$ with comparison constant $C>0$ if

$$
\frac{1}{C} Q_{1} \leq Q_{2} \leq C Q_{1}
$$

\section{Background}

In this paper we only consider complete, locally compact, geodesic metric spaces. We provide the relevant definitions of the notions used in this note. In what follows, given a metric space $(Z, d), z \in Z$ and $r>0$, we set $B(z, r):=\{x \in Z: d(x, z)<r\}$ and $\bar{B}(z, r):=\{x \in Z: d(x, z) \leq r\}$.

Definition 2.1. A complete unbounded locally compact geodesic metric space $(Z, d)$ is said to be Gromov hyperbolic if there exists $\delta \geq 0$ such that whenever $x, y, z \in Z$ and $[x, y],[y, z],[z, x]$ are geodesic paths in $Z$ with end points $x, y$, end points $y, z$, and end points $z, x$, respectively, then

$$
[x, y] \subset \bigcup_{w \in[y, z] \cup[z, x]} \bar{B}(w, \delta) .
$$

Here, if $\delta=0$, we interpret $\bar{B}(w, \delta)$ to be the set $\{w\}$.

The above definition of Gromov hyperbolic space is from [BHK], but readers might want to keep in mind that there are alternate definitions of Gromov hyperbolic spaces in literature that do not require the metric space to be geodesic or locally compact, see for example [BuS, Definition 1.2.2, Proposition 2.1.2, Proposition 2.1.3].

Definition 2.2. We say that a Gromov hyperbolic space $(Z, d)$ is roughly starlike (or, $M$-roughly starlike) if there exists $M \geq 0$ and $z_{0} \in Z$ such that for all $z \in Z$ there is a geodesic ray $\gamma:[0, \infty) \rightarrow Z$ with $\gamma(0)=z_{0}$ and $t_{0} \in[0, \infty)$ such that $d\left(z, \gamma\left(t_{0}\right)\right) \leq M$.

Trees with each vertex of degree at least 2 are Gromov hyperbolic with $\delta=0$ and are roughly starlike with $M=0$. Uniform domains, equipped with the quasihyperbolic metric, are necessarily Gromov hyperbolic and roughly starlike, see the discussion in [BHK, Chapter 3]. The example

$$
X=[0, \infty) \cup \bigcup_{n \in \mathbf{N}}[n, n+\sqrt{-1} n] \subset \mathbf{C},
$$

equipped with the length metric induced by the Euclidean metric shows that there are Gromov hyperbolic spaces that are not roughly starlike.

Following [BHK], for each $\varepsilon>0$ we consider uniformization of Gromov hyperbolic spaces with parameter $\varepsilon$.

Definition 2.3. Let $(Z, d)$ be a Gromov hyperbolic space, $z_{0} \in Z$, and $\varepsilon>0$. We consider the "density" function $\rho_{\varepsilon}^{Z}: Z \rightarrow(0,1]$ given by

$$
\rho_{\varepsilon}^{Z}(z):=e^{-\varepsilon d\left(z, z_{0}\right)} .
$$

This density function induces a metric on $Z$, given by

$$
d_{\varepsilon}\left(z_{1}, z_{2}\right)=\inf _{\gamma} \int_{\gamma} \rho_{\varepsilon}^{Z} d s
$$

for $z_{1}, z_{2} \in Z$, where the infimum is over all rectifiable paths $\gamma$ in $Z$ with end points $z_{1}$ and $z_{2}$ and the integral is taken with respect to the arc-length measure $d s$. We 
denote this induced metric space $\left(Z, d_{\varepsilon}\right)$ by $Z_{\varepsilon}$. Furthermore, for a locally rectifiable path $\gamma$ in $Z$, we denote by $\ell(\gamma)$ its length with respect to the metric $d$, and by $\ell_{\varepsilon}(\gamma)$ its length with respect to the metric $d_{\varepsilon}$. It is not difficult to see that

$$
\ell_{\varepsilon}(\gamma)=\int_{\gamma} \rho_{\varepsilon}^{Z} d s
$$

The above construction of uniformization is from [BHK, Chapter 4]. As mentioned above, from [BHK] we know that if $Z$ is Gromov hyperbolic and $\varepsilon \leq \varepsilon_{0}=\varepsilon_{0}(\delta)$, then $Z_{\varepsilon}$ is a uniform domain, that is, it satisfies the following definition.

Definition 2.4. Let $Z$ be a locally complete, non-complete metric space, and set $\partial Z:=\bar{Z} \backslash Z$. We say that $Z$ is a uniform domain (or a uniform space) if there is a constant $\lambda \geq 1$ such that for each pair of points $x, y \in Z$ there is a rectifiable curve $\gamma$ in $Z$ with end points $x$ and $y$ satisfying

(1) $\ell(\gamma) \leq \lambda d(x, y)$, that is, $\gamma$ is $\lambda$-quasiconvex,

(2) for each $z \in \gamma$,

$$
\delta_{Z}(z):=\operatorname{dist}(z, \partial Z) \geq \lambda^{-1} \min \{\ell(\gamma(x, z)), \ell(\gamma(z, y))\} .
$$

Here $\gamma(x, z)$ is any of the subcurves of $\gamma$ with end points $x, z$. The number $\lambda$ is called a uniformity constant of $Z$, and a curve $\gamma$ satisfying the two listed conditions above is said to be a uniform curve or a $\lambda$-uniform curve.

From [MS, GeO, BHK], there is a natural deformation of the metric on a uniform domain $(Z, d)$, called the quasihyperbolic metric.

Definition 2.5. Given a locally compact, non-complete metric space $(Z, d)$, the quasihyperbolic metric $k$ on $Z$ is given by

$$
k(x, y)=\inf _{\gamma} \int_{\gamma} \frac{1}{\delta_{Z}(\gamma(t))} d s(t)
$$

when $x, y \in Z$. Here the infimum is over all rectifiable curves $\gamma$ in $Z$ with end points $x$ and $y$, and $d s$ is the arc-length measure.

We assume from now on that $(X, d)$ and $(Y, d)$ are Gromov hyperbolic spaces.

Definition 2.6. A map $\Phi: Y \rightarrow X$ is a $\tau$-rough isometry if

$$
d\left(y_{1}, y_{2}\right)-\tau \leq d\left(\Phi\left(y_{1}\right), \Phi\left(y_{2}\right)\right) \leq d\left(y_{1}, y_{2}\right)+\tau
$$

for all $y_{1}, y_{2} \in Y$ and $\Phi(Y)$ is $\tau$-dense in $X$, that is, for each $x \in X$ there is some $y_{x} \in Y$ such that $d\left(x, \Phi\left(y_{x}\right)\right) \leq \tau$.

Note that we do not require $\Phi$ to be continuous, and we do not require it to be injective or surjective.

Lemma 2.7. Given a $\tau$-rough isometry $\Phi: Y \rightarrow X$, there exists a $3 \tau$-rough isometry $\Phi^{-1}: X \rightarrow Y$ such that for all $y \in Y$ and $x \in X$ we have

$$
d\left(y, \Phi^{-1}(\Phi(y))\right) \leq 2 \tau, \quad d\left(x, \Phi\left(\Phi^{-1}(x)\right)\right) \leq \tau .
$$

This seems to be well-known (see for example [BS], [Bow, Proposition 3.2]), but as we were not able to find a published proof of this fact, we provide the proof here for the convenience of the reader.

Proof. We first construct $\Phi^{-1}: X \rightarrow Y$ as follows. Given $x \in X$, by the fact that every point in $X$ is within a distance $\tau$ of $\Phi(Y)$, we can find a point $y_{x} \in Y$ such 
that $d\left(\Phi\left(y_{x}\right), x\right) \leq \tau$. We choose one such $y_{x}$ and set $\Phi^{-1}(x)=y_{x}$. Note that

$$
d\left(\Phi\left(\Phi^{-1}(x)\right), x\right)=d\left(\Phi\left(y_{x}\right), x\right) \leq \tau .
$$

Moreover, for $y \in Y$, with the choice of $x=\Phi(y)$, we have the point $y_{\Phi(y)}$ as a point in $Y$ that $\Phi^{-1}$ maps $x$ to. Then $d\left(\Phi\left(y_{\Phi(y)}\right), x\right) \leq \tau$, and so

$$
d\left(\Phi^{-1}(\Phi(y)), y\right)=d\left(y_{\Phi(y)}, y\right) \leq \tau+d\left(\Phi\left(y_{\Phi(y)}\right), \Phi(y)\right) \leq 2 \tau .
$$

For $x, x^{\prime} \in X$, we have

$$
\begin{aligned}
d\left(\Phi^{-1}(x), \Phi^{-1}\left(x^{\prime}\right)\right)=d\left(y_{x}, y_{x^{\prime}}\right) & \leq \tau+d\left(\Phi\left(y_{x}\right), \Phi\left(y_{x^{\prime}}\right)\right) \\
& \leq \tau+d\left(\Phi\left(y_{x}\right), x\right)+d\left(x, x^{\prime}\right)+d\left(x^{\prime}, \Phi\left(y_{x^{\prime}}\right)\right) \\
& \leq 3 \tau+d\left(x, x^{\prime}\right) .
\end{aligned}
$$

Furthermore,

$$
\begin{aligned}
d\left(\Phi^{-1}(x), \Phi^{-1}\left(x^{\prime}\right)\right)=d\left(y_{x}, y_{x^{\prime}}\right) & \geq d\left(\Phi\left(y_{x}\right), \Phi\left(y_{x^{\prime}}\right)\right)-\tau \\
& \geq-d\left(\Phi\left(y_{x}\right), x\right)+d\left(x, x^{\prime}\right)-d\left(x^{\prime}, \Phi\left(y_{x^{\prime}}\right)\right)-\tau \\
& \geq d\left(x, x^{\prime}\right)-3 \tau .
\end{aligned}
$$

Finally, given $y \in Y$, we set $x=\Phi(y)$ and note from the first part of the argument that

$$
d\left(y, \Phi^{-1}(x)\right)=d\left(y, \Phi^{-1}(\Phi(y))\right) \leq 2 \tau .
$$

This concludes the proof.

Remark 2.8. Note that if $\Phi$ is a $\tau$-rough isometry, then it is also a $3 \tau$-rough isometry. Hence, by replacing $\tau$ with $3 \tau$ if necessary, we will assume in the rest of the paper that both $\Phi$ and $\Phi^{-1}$ are $\tau$-rough isometries with

$$
d\left(y, \Phi^{-1}(\Phi(y))\right) \leq \tau \quad \text { and } \quad d\left(x, \Phi\left(\Phi^{-1}(x)\right)\right) \leq \tau .
$$

Remark 2.9. Suppose that $X$ and $Y$ are two geodesic metric spaces and $\Phi: Y \rightarrow$ $X$ is a $\tau$-rough isometry. From [BH, Proposition 1.22] we know that if $X$ is $\delta$-Gromov hyperbolic, then the Gromov product $(x \mid y)_{y_{0}}, x, y \in Y$, satisfies the so-called $6 \delta$ inequality:

$$
(x \mid y)_{y_{0}} \geq \min \left\{(x \mid z)_{y_{0}},(z \mid y)_{y_{0}}\right\}-6 \delta,
$$

where the Gromov product is defined by

$$
(x \mid y)_{y_{0}}:=\frac{1}{2}\left[d\left(x, y_{0}\right)+d\left(y, y_{0}\right)-d(x, y)\right] .
$$

Moreover, if $Y$ is a geodesic space and satisfies the above $6 \delta$-inequality, then $Y$ is $36 \delta$-Gromov hyperbolic. From the above it follows immediately that if $Y$ is $\delta$-Gromov hyperbolic, then $X$ is $6(3 \tau+6 \delta)$-Gromov hyperbolic.

If $Y$ is both $\delta$-Gromov hyperbolic and $M$-roughly starlike, then $X$ is $M^{\prime}(\delta, \tau, M)$ roughly starlike. To see this, note that if $x \in X$, then setting $y=\Phi^{-1}(x)$, by the rough starlikeness of $Y$ there is a geodesic ray $\gamma:[0, \infty) \rightarrow Y$ with $\gamma(0)=y_{0}$ and some $t_{0} \geq 0$ such that $d\left(\gamma\left(t_{0}\right), y\right) \leq M$. For $k=0,1, \cdots$ let $b_{k}=\Phi(\gamma(k(1+\tau)))$ and $w=\Phi\left(\gamma\left(t_{0}\right)\right)$. Then $d(x, w) \leq d\left(y, \gamma\left(t_{0}\right)\right)+\tau \leq M+\tau$, and $d\left(w, b_{i}\right) \leq d\left(\gamma\left(t_{0}\right), \gamma(i(1+\right.$ $\tau)))+\tau \leq 1+2 \tau$ for some $i \in \mathbf{N}$. By the geodesic stability result [BuS, Theorem 1.3.2 of page 5], together with an invocation of the Arzelà-Ascoli theorem there is a positive number $h(\tau, \delta)$ and a geodesic ray $\beta:[0, \infty) \rightarrow X$ with $\beta(0)=x_{0}$ and $s_{0} \geq 0$ such that $d\left(b_{i}, \beta\left(s_{0}\right)\right) \leq h(\tau, \delta)$. Combining these together, we get

$$
d\left(x, \beta\left(s_{0}\right)\right) \leq M+\tau+1+2 \tau+h(\tau, \delta)=1+M+3 \tau+h(\tau, \delta),
$$


that is, $X$ is $M^{\prime}(\delta, \tau, M)$-roughly starlike with

$$
M^{\prime}(\delta, \tau, M)=1+M+3 \tau+h(\tau, \delta) .
$$

Interestingly, the geodesic stability property mentioned above also characterizes Gromov hyperbolicity, see [Bo]. The result [Bo, Proposition 3.1] together with the fact that the path in $X$ obtained by concatenating the geodesic segments connecting $b_{k}, b_{k+1}, k=0,1, \cdots$ is a $(\lambda, \tau \lambda)$-chord-arc curve in the sense defined in [Bo, Page 295] gives a more explicit estimate for $h(\tau, \delta)$ than that found in [BuS]. Here,

$$
\lambda=\frac{1+2 \tau}{1+\tau} .
$$

There is a more general notion of Gromov hyperbolicity that does not require the space to be a geodesic space, and this notion is given with respect to the Gromov product; see for example [Gr, GH]. Since what we do with path integrals requires our space to be a geodesic space, we do not consider the Gromov product definition of hyperbolicity.

Remark 2.10. The density $\rho_{\varepsilon}^{Z}$ as considered in Definition 2.3 is an example of a large class of densities, called conformal densities, used to deform metrics on a given metric space, see for example [KL, BKR]. A positive continuous function $\rho$ on a metric space $Z$ is a Harnack conformal density if there is a constant $A \geq 1$ such that whenever $x, y \in X$ with $d(x, y) \leq 1$, we have

$$
\frac{1}{A} \leq \frac{\rho(x)}{\rho(y)} \leq A
$$

The nomenclature is justified by the fact that if $\rho$ is a conformal density on $(Z, d)$ and the metric on $Z$ is modified to a new metric $d_{\rho}$ according to the scheme given in Definition 2.3 with $\rho$ playing the role of $\rho_{\varepsilon}^{Z}$, then the natural identity map $\operatorname{Id}:(Z, d) \rightarrow$ $\left(Z, d_{\rho}\right)$ is a (metrically) 1-quasiconformal map. The usage of "Harnack" in the above nomenclature echoes the Harnack property of positive harmonic functions.

We fix two distinguished points $x_{0} \in X$ and $y_{0} \in Y$. We are concerned with the two densities

$$
\rho_{\varepsilon}^{X}(x)=e^{-\varepsilon d\left(x_{0}, x\right)} \quad \text { and } \quad \rho_{\varepsilon}^{Y}(y)=e^{-\varepsilon d\left(y_{0}, y\right)} .
$$

We denote by $X_{\varepsilon}$ and $Y_{\varepsilon}$ the $\varepsilon$-uniformizations of $X$ and $Y$.

Remark 2.11. Given a conformal density $\rho$ on $Z$ as in (2), and $Z$ a geodesic space, we see that whenever $K \in \mathbf{N}$ and $x, y \in X$ such that $d(x, y) \leq K$, then

$$
\frac{1}{A^{K}} \leq \frac{\rho(x)}{\rho(y)} \leq A^{K}
$$

Note that by the triangle inequality,

$$
\frac{\rho_{\varepsilon}^{X}(x)}{\rho_{\varepsilon}^{X}(y)}=e^{-\varepsilon\left[d\left(x, x_{0}\right)-d\left(y, x_{0}\right)\right]} \geq e^{-\varepsilon d(x, y)} \geq e^{-\varepsilon}
$$

when $d(x, y) \leq 1$. Similarly, we get

$$
\frac{\rho_{\varepsilon}^{X}(x)}{\rho_{\varepsilon}^{X}(y)}=e^{-\varepsilon\left[d\left(x, x_{0}\right)-d\left(y, x_{0}\right)\right]} \leq e^{\varepsilon d(x, y)} \leq e^{\varepsilon} .
$$

Thus both $\rho_{\varepsilon}^{X}$ and $\rho_{\varepsilon}^{Y}$ satisfy (2) with $A=e^{\varepsilon}$. 
As described above, a given roughly starlike Gromov hyperbolic space can be uniformized and then the resulting space can be equipped with its quasihyperbolic metric (see (1) above for the definition of quasihyperbolic metric). The outcome may not be isometric to the original Gromov hyperbolic space, but as the next lemma shows, it is biLipschitz equivalent.

Lemma 2.12. Let $(X, d)$ be a roughly starlike Gromov hyperbolic space and $\varepsilon>0$. Then $\left(X_{\varepsilon}, k\right)$ is biLipschitz equivalent to $(X, d)$.

In the above lemma, $k$ is the quasihyperbolic metric with respect to the uniformized space $X_{\varepsilon}$. Note that we do not assume any condition on $\varepsilon$ apart from that it is positive. The above lemma was proved in [BHK, Proposition 4.37] for the setting where $\varepsilon \leq \varepsilon_{0}$. For the convenience of the reader, we provide a short proof of Lemma 2.12 here.

Proof. Let $\delta_{\varepsilon}$ be the distance to the boundary $\partial X_{\varepsilon}:=\overline{X_{\varepsilon}} \backslash X_{\varepsilon}$. Recall that we have a distinguished point $x_{0} \in X$ in the definition of $X_{\varepsilon}$. Note that the quasihyperbolic distance $k$ is given by

$$
k(x, y)=\inf _{\gamma} \int_{\gamma} \frac{1}{\delta_{\varepsilon}(\gamma(t))} d s_{\varepsilon}(t),
$$

where we took $\gamma$ to be arc-length parametrized with respect to the metric $d$ on $X$ with end points $x$ and $y$, and $d s_{\varepsilon}$ is the arc-length measure with arc-lengths computed with respect to the uniformized metric $d_{\varepsilon}$. By the construction of uniformization, we have that $d s_{\varepsilon}(z)=e^{-\varepsilon d\left(z, x_{0}\right)} d s$. On the other hand, by a straightforward calculation (see Lemma 3.4 below), we know that $\delta_{\varepsilon}(z) \simeq e^{-\varepsilon d\left(z, x_{0}\right)}$. It follows that

$$
k(x, y) \simeq \inf _{\gamma} \ell(\gamma)=d(x, y)
$$

Remark 2.13. The flip side of the above lemma is the following question. Suppose that $Z$ is a uniform space and $X=(Z, k)$ the metric space obtained by considering the quasihyperbolic metric on $Z$. From $[\mathrm{BHK}]$ we know that $X$ is then Gromov hyperbolic. Is there a choice of $\varepsilon>0$ such that $X_{\varepsilon}$ is biLipschitz equivalent to $Z$ ? We do not know at this time whether such a choice of $\varepsilon$ always exists. The difficulty underlying this question stems from the problem that the uniformization of two Gromov hyperbolic spaces that are biLipschitz equivalent need not result in two biLipschitz equivalent metric spaces; uniformization is a more complex process than quasihyperbolization.

On the other hand, from [GeO, Corollary 1], $\Omega$ is a uniform domain if and only if the quasihyperbolic metric $k$ is equivalent to the metric given by

$$
j(x, y):=\log \left(1+\frac{d(x, y)}{\delta_{Z}(x) \wedge \delta_{Z}(y)}\right) .
$$

Using the metric $j$ rather than $k$ to perform the uniformization procedure does result in biLipschitz equivalence.

\section{Results}

Recall that $X$ and $Y$ are Gromov hyperbolic spaces and $\Phi: Y \rightarrow X$ is a $\tau$-rough isometry with a $\tau$-rough isometric inverse (in the sense of Remark 2.8) such that $\Phi\left(y_{0}\right)=x_{0}$ with $x_{0} \in X$ and $y_{0} \in Y$. In what follows, all curves are assumed to be parametrized by (hyperbolic) arclength unless otherwise specified. 
Lemma 3.1. Suppose that $\rho: Y \rightarrow(0, \infty)$ satisfies the Harnack condition (2) with constant $A$. Let $L>1$ and $\gamma:[0, L] \rightarrow Y$ be a curve with $\ell(\gamma)=L$. Choose $N \in \mathbf{N}$ such that $N \leq L<N+1$. Then

$$
\int_{\gamma} \rho d s \simeq \sum_{i=0}^{N-1} \rho\left(a_{i}\right)
$$

where $a_{i}=\gamma(i q)$ with $q:=\frac{L}{N}$. The comparison constant in (3) can be taken to be $2 A^{2}$. If $L \leq Q$ with $Q \geq 1$ a fixed number, we instead have $\int_{\gamma} \rho d s \simeq L \cdot \rho(\gamma(0))$ with comparison constant $A^{Q+1}$.

Proof. The statement dealing with the case $L \leq Q$ is clear as $\rho$ satisfies the Harnack condition; hence, we will only consider the case $L \geq 1$.

Note that $1 \leq q<2$. For $0 \leq i \leq N-1$, let $\gamma_{i}:[0, q] \rightarrow Y$ be the curve given by $\gamma_{i}(t)=\gamma(i q+t)$. Note that $\gamma_{i}$ is parametrized by arclength because $\gamma$ is. Hence the length $\ell\left(\gamma_{i}\right)$ of $\gamma_{i}$ satisfies $1 \leq \ell\left(\gamma_{i}\right)<2$. By condition $(2)$, it follows that

$$
\frac{1}{A^{2}} \rho\left(a_{i}\right) \leq \int_{\gamma_{i}} \rho d s \leq 2 A^{2} \rho\left(a_{i}\right) \text {. }
$$

Hence

$$
\sum_{i=0}^{N-1} \rho\left(a_{i}\right) \simeq \sum_{i=0}^{N-1} \int_{\gamma_{i}} \rho d s=\int_{\gamma} \rho d s
$$

with comparison constant $2 A^{2}$.

Remark 3.2. Lemma 3.1 holds in $X$ as well.

Lemma 3.3. Suppose $x, y \in Y$ with $d(x, y)>1$. Let $L>1$ and $\gamma:[0, L] \rightarrow Y$ be a curve with $\gamma(0)=x$ and $\gamma(L)=y$. Fix $N \in \mathbf{N}$ such that $N \leq L<N+1$. Then,

$$
\int_{\gamma} \rho_{\varepsilon}^{Y} d s \simeq \sum_{i=0}^{N-1} \rho_{\varepsilon}^{X}\left(\Phi\left(a_{i}\right)\right) \simeq\left(\sum_{i=0}^{N-2} \rho_{\varepsilon}^{X}\left(\Phi\left(a_{i}\right)\right)\right)+\rho_{\varepsilon}^{X}(\Phi(y))
$$

where $q=\frac{L}{N}$ and $a_{i}=\gamma(i q)$ for $0 \leq i \leq N$. In the above, we adopt the convention that $\sum_{i=0}^{N-2} \rho_{\varepsilon}^{X}\left(\Phi\left(a_{0}\right)\right)=0$ if $N=1$. The comparison constants depend solely on $\varepsilon$ and $\tau$.

Proof. Note that $a_{0}=x$ and $a_{N}=y$. For $0 \leq i \leq N$ let $b_{i}=\Phi\left(a_{i}\right)$. By Lemma 3.1,

$$
\int_{\gamma} \rho_{\varepsilon}^{Y} d s \simeq \sum_{i=0}^{N-1} \rho_{\varepsilon}^{Y}\left(a_{i}\right)
$$

with comparison constant $2 e^{2 \varepsilon}$. Now, $\rho_{\varepsilon}^{Y}\left(a_{i}\right)=e^{-\varepsilon d\left(y_{0}, a_{i}\right)}$ and, as $\Phi$ is a $\tau$-rough isometry, we have

$$
d\left(y_{0}, a_{i}\right)-\tau \leq d\left(x_{0}, b_{i}\right) \leq d\left(y_{0}, a_{i}\right)+\tau .
$$

In particular,

$$
e^{-\tau \varepsilon} \leq \frac{\rho_{\varepsilon}^{Y}\left(a_{i}\right)}{\rho_{\varepsilon}^{X}\left(b_{i}\right)} \leq e^{\tau \varepsilon}
$$

for all $i$. Hence we have

$$
\sum_{i=0}^{N-1} \rho_{\varepsilon}^{Y}\left(a_{i}\right) \simeq \sum_{i=0}^{N-1} \rho_{\varepsilon}^{X}\left(\Phi\left(a_{i}\right)\right)
$$


with comparison constant $e^{\tau \varepsilon}$. Hence

$$
\int_{\gamma} \rho_{\varepsilon}^{Y} d s \simeq \sum_{i=0}^{N-1} \rho_{\varepsilon}^{X}\left(\Phi\left(a_{i}\right)\right)
$$

with comparison constant $2 e^{2 \varepsilon+\tau \varepsilon}$.

The second comparability follows as $d\left(a_{N-1}, y\right) \leq 2$, and so $\rho_{\varepsilon}^{Y}\left(a_{N-1}\right) \simeq \rho_{\varepsilon}^{Y}(y)$ with comparison constant $e^{2 \varepsilon}$, see Remark 2.11.

Lemma 3.4. Let $Y$ be a Gromov hyperbolic space and $\varepsilon>0$. Then for each $x \in Y$ we have

$$
\delta_{\varepsilon}(x):=\operatorname{dist}\left(x, \partial Y_{\varepsilon}\right):=\operatorname{dist}\left(x, \overline{Y_{\varepsilon}} \backslash Y_{\varepsilon}\right) \gtrsim e^{-\varepsilon d\left(x, y_{0}\right)}=\rho_{\varepsilon}^{Y}(x),
$$

with comparison constant $1 / \varepsilon$. If in addition $Y$ is an $M$-roughly starlike space, then

$$
\delta_{\varepsilon}(x) \simeq \rho_{\varepsilon}^{Y}(x)
$$

with comparison constant $\left[M+\varepsilon^{-1}\right] e^{\varepsilon M}$.

Proof. Let $x \in Y$ and $\gamma$ be any path from $x$ that leaves every compact subset of $Y$. Then we have

$$
\int_{\gamma} e^{-\varepsilon d\left(\gamma(t), y_{0}\right)} d t \geq \int_{0}^{\infty} e^{-\varepsilon\left[d\left(y_{0}, x\right)+t\right]} d t=\frac{e^{-\varepsilon d\left(y_{0}, x\right)}}{\varepsilon} .
$$

Taking the infimum over all such $\gamma$ gives

$$
\delta_{\varepsilon}(x) \geq \frac{\rho_{\varepsilon}^{Y}(x)}{\varepsilon} .
$$

Now suppose that $Y$ is also $M$-roughly starlike. Let $x \in Y$ and $\gamma:[0, \infty) \rightarrow Y$ be a geodesic ray from $y_{0}$ so that there is some $t_{0} \in[0, \infty)$ for which $d\left(x, \gamma\left(t_{0}\right)\right) \leq M$. Let $\beta$ be a geodesic with end points $x$ and $\gamma\left(t_{0}\right)$; then the concatenation $\gamma_{*}$ of $\left.\gamma\right|_{\left[t_{0}, \infty\right)}$ and $\beta$ gives us that

$$
\delta_{\varepsilon}(x) \leq \int_{\gamma_{*}} e^{-\varepsilon d\left(\gamma_{*}(t), y_{0}\right)} d t .
$$

Note that for points $w \in \beta, d\left(x, y_{0}\right)-M \leq d\left(w, y_{0}\right) \leq d\left(x, y_{0}\right)+M$, and so

$$
\delta_{\varepsilon}(x) \leq M e^{\varepsilon M} e^{-\varepsilon d\left(x, y_{0}\right)}+\int_{t_{0}}^{\infty} e^{-\varepsilon t} d t \leq M e^{\varepsilon M} e^{-\varepsilon d\left(x, y_{0}\right)}+\varepsilon^{-1} e^{-\varepsilon t_{0}} .
$$

Moreover, $t_{0}=d\left(\gamma\left(t_{0}\right), y_{0}\right) \geq d\left(y_{0}, x\right)-M$. Therefore

$$
\delta_{\varepsilon}(x) \leq\left[M+\varepsilon^{-1}\right] e^{\varepsilon M} e^{-\varepsilon d\left(y_{0}, x\right)} .
$$

It follows that

$$
\delta_{\varepsilon}(z) \simeq e^{-\varepsilon d\left(z, y_{0}\right)},
$$

with comparison constant $\left[M+\varepsilon^{-1}\right] e^{\varepsilon M}$.

In the proof of the following lemma we use $\Phi^{-1}$ together with $\Phi$, see Lemma 2.7 regarding the construction of $\Phi^{-1}$.

Lemma 3.5. Let $X$ and $Y$ be two Gromov hyperbolic spaces and $\Phi: Y \rightarrow X$ be a $\tau$-rough isometry. Then for $\varepsilon>0$ and for each $y \in Y$,

$$
\delta_{\varepsilon}(y) \simeq \delta_{\varepsilon}(\Phi(y))
$$

with the comparison constant depending solely on $\varepsilon$ and $\tau$. 
Proof. Let $y \in Y$ and $x:=\Phi(y)$. Let $\gamma:[0, \infty) \rightarrow Y$ be any path from $y$ that leaves every compact subset of $Y$. Set $a_{0}:=y$ and for $k \in \mathbf{N}$ let $a_{k}:=\gamma((1+\tau) k)$. Recall the definition of $\ell_{\varepsilon}(\gamma)$ from Definition 2.3. Then a simple modification of Lemma 3.1 together with the rough isometric equivalence of $X$ and $Y$ tells us that

$$
\ell_{\varepsilon}(\gamma) \simeq \sum_{k=0}^{\infty} \rho_{\varepsilon}^{Y}\left(a_{k}\right) \simeq \sum_{k=0}^{\infty} \rho_{\varepsilon}^{X}\left(\Phi\left(a_{k}\right)\right) .
$$

Let $\beta_{k}$ be a hyperbolic geodesic in $X$ with end points $\Phi\left(a_{k}\right)$ and $\Phi\left(a_{k+1}\right)$ and $\beta$ be the concatenation of the paths $\beta_{k}, k=0,1, \cdots$. Since $\ell\left(\left.\gamma\right|_{[k, k+1]}\right)=1+\tau$, it follows that $1 \leq d\left(\Phi\left(a_{k}\right), \Phi\left(a_{k+1}\right)\right) \leq 1+2 \tau$; therefore $1 \leq \ell\left(\beta_{k}\right) \leq 1+2 \tau$. Therefore by the second part of Lemma 3.1 and the above estimate,

$$
\ell_{\varepsilon}(\beta)=\sum_{k=0}^{\infty} \ell_{\varepsilon}\left(\beta_{k}\right) \simeq \sum_{k=0}^{\infty} \rho_{\varepsilon}^{X}\left(\Phi\left(a_{k}\right)\right) \simeq \ell_{\varepsilon}(\gamma) .
$$

Note that as $\Phi$ is a rough isometry and $\gamma$ leaves every bounded subset of the proper space $Y$, the path $\beta$ also leaves every bounded subset of $X$. Since we require in this paper that $X$ and $Y$ are locally compact geodesic spaces, we know from the Hopf-Rinow theorem that $X_{\varepsilon}$ and $Y_{\varepsilon}$ are length spaces. Hence, taking the infimum over all $\gamma:[0, \infty) \rightarrow Y$ with $\gamma(0)=y$ as above yields

$$
\ell_{\varepsilon}(\beta) \lesssim \delta_{\varepsilon}(y)
$$

It follows that

$$
\delta_{\varepsilon}(\Phi(y)) \leq \ell_{\varepsilon}(\beta) \lesssim \delta_{\varepsilon}(y) .
$$

Reversing the roles of $X$ and $Y$, and replacing $\Phi$ with $\Phi^{-1}$ gives

$$
\delta_{\varepsilon}\left(\Phi^{-1}(\Phi(y))\right) \lesssim \delta_{\varepsilon}(\Phi(y)) .
$$

Since $d\left(y, \Phi^{-1}(\Phi(y))\right) \leq \tau$, it follows from Lemma 3.4 and the second part of Lemma 3.1 that

$$
\begin{aligned}
\delta_{\varepsilon}(y) & \leq \delta_{\varepsilon}\left(\Phi^{-1}(\Phi(y))\right)+d_{\varepsilon}\left(y, \Phi^{-1}(\Phi(y))\right) \\
& \lesssim \delta_{\varepsilon}\left(\Phi^{-1}(\Phi(y))\right)+\tau \rho_{\varepsilon}^{Y}\left(\Phi^{-1}(\Phi(y))\right) \lesssim \delta_{\varepsilon}\left(\Phi^{-1}(\Phi(y))\right) .
\end{aligned}
$$

Lemma 3.6. Let $x, y \in Y$ be such that $d(x, y) \leq 4+\tau$, and let $\gamma$ be a hyperbolic geodesic in $Y$ with end points $x, y$. Then

$$
\ell_{\varepsilon}(\gamma) \simeq d_{\varepsilon}(x, y) \simeq e^{-\varepsilon d\left(x, y_{0}\right)} d(x, y)
$$

and $\gamma$ is a uniform curve with respect to the metric $d_{\varepsilon}$ on $Y_{\varepsilon}$, with uniformity constant depending only on $\varepsilon$, and $\tau$.

Proof. Recall from Definition 2.3 that the length $\ell_{\varepsilon}(\gamma)$ of $\gamma$ in the uniformized metric $d_{\varepsilon}$ is given by

$$
\ell_{\varepsilon}(\gamma)=\int_{\gamma} e^{-\varepsilon d\left(\gamma(t), y_{0}\right)} d t
$$

and as

$d\left(x, y_{0}\right)-4-\tau \leq d\left(x, y_{0}\right)-d(x, z) \leq d\left(y_{0}, z\right) \leq d\left(x, y_{0}\right)+d(x, z) \leq d\left(x, y_{0}\right)+4+\tau$.

for each $z$ in the trajectory of $\gamma$, we see that

$$
\ell(\gamma) e^{-\varepsilon d\left(x, y_{0}\right)} e^{-\varepsilon(4+\tau)} \leq \ell_{\varepsilon}(\gamma) \leq \ell(\gamma) e^{-\varepsilon d\left(x, y_{0}\right)} e^{\varepsilon(4+\tau)} .
$$


Observe that $d(x, y)=\ell(\gamma)$. On the other hand, with $\beta$ any rectifiable non-geodesic curve in $Y$ with end points $x$ and $y$, we must have $\ell(\beta)>d(x, y)$, and so with $t_{0} \in[0, \ell(\beta)]$ the smallest number for which $d\left(x, \beta\left(t_{0}\right)\right)=d(x, y)$, we get

$$
\int_{\beta} \rho_{\varepsilon}^{Y} d s \geq \int_{0}^{t_{0}} \rho_{\varepsilon}^{Y} \circ \beta(t) d t \geq d(x, y) e^{-\varepsilon d\left(x, y_{0}\right)} e^{-\varepsilon(4+\tau)} .
$$

Therefore

$$
d(x, y) e^{-\varepsilon d\left(x, y_{0}\right)} e^{\varepsilon(4+\tau)} \geq \ell_{\varepsilon}(\gamma) \geq d_{\varepsilon}(x, y)=\inf _{\beta} \int_{\beta} \rho_{\varepsilon}^{Y} d s \geq d(x, y) e^{-\varepsilon d\left(x, y_{0}\right)} e^{-\varepsilon(4+\tau)} .
$$

It immediately follows that

$$
d_{\varepsilon}(x, y) \simeq d(x, y) e^{-\varepsilon d\left(x, y_{0}\right)},
$$

and consequently, we have that $\ell_{\varepsilon}(\gamma) \lesssim d_{\varepsilon}(x, y)$, that is, $\gamma$ is a $C$-quasiconvex curve in $Y_{\varepsilon}$, with constant $C$ depending only on $\varepsilon$ and $\tau$. Recall that a curve is a $C$ quasiconvex if its length is dominated by at most $C$ times the distance between the endpoints of the curve (see Definition 2.4). Moreover, from Lemma 3.4 and the fact that $d(x, y) \leq 4+\tau$ we know that for $z \in \gamma$,

$$
\delta_{\varepsilon}(z) \gtrsim e^{-\varepsilon d\left(z, y_{0}\right)} \gtrsim e^{-\varepsilon d\left(x, y_{0}\right)} \gtrsim \ell_{\varepsilon}(\gamma),
$$

that is, $\gamma$ is a uniform curve, with a uniformity constant that depend only on $\varepsilon$ and $\tau$.

From the above lemma, to show that $Y_{\varepsilon}$ is a uniform domain it suffices to show that $x, y \in Y$ can be connected by a uniform curve when $d(x, y) \geq 4+\tau$. This is the focus of the remaining discussion.

Lemma 3.7. Let $x, y \in Y$ be such that $d(x, y) \geq 2+\tau$. Then

$$
d_{\varepsilon}(x, y) \simeq d_{\varepsilon}(\Phi(x), \Phi(y)) .
$$

See Lemma 2.7 regarding the construction of $\Phi^{-1}$.

Proof. Let $\gamma:[0, L] \rightarrow Y$ be any curve with $\gamma(0)=x, \ell(\gamma)=L$, and $\gamma(L)=y$. Note that $L \geq 2+\tau \geq 2$. We fix $N \in \mathbf{N}$ such that $N \leq L<N+1$. Let $q=\frac{L}{N}$ and, for $0 \leq i \leq N$, let $a_{i}=\gamma(i q)$ and $b_{i}=\Phi\left(a_{i}\right)$. Then $d\left(b_{i}, b_{i+1}\right) \leq d\left(a_{i}, a_{i+1}\right)+\tau \leq 4+\tau$, and so by Lemma 3.6 we have

$$
d_{\varepsilon}\left(b_{i}, b_{i+1}\right) \lesssim e^{-\varepsilon d\left(b_{i}, x_{0}\right)}=\rho_{\varepsilon}^{X}\left(b_{i}\right)
$$

with comparability constant depending only on $\varepsilon$ and $\tau$. It follows that

$$
d_{\varepsilon}(\Phi(x), \Phi(y)) \leq \sum_{i=0}^{N-1} d_{\varepsilon}\left(b_{i}, b_{i+1}\right) \lesssim \sum_{i=0}^{N-1} \rho_{\varepsilon}^{X}\left(b_{i}\right) .
$$

By Lemma 3.3, we have $\sum_{i=0}^{N-1} \rho_{\varepsilon}^{X}\left(b_{i}\right) \simeq \int_{\gamma} \rho_{\varepsilon}^{Y} d s$. Infimizing over all paths $\gamma$ connecting $x$ to $y$ yields

$$
d_{\varepsilon}(\Phi(x), \Phi(y)) \lesssim \inf _{\gamma} \int_{\gamma} \rho_{\varepsilon}^{Y} d s=d_{\varepsilon}(x, y) .
$$

Next, note that $d(\Phi(x), \Phi(y)) \geq d(x, y)-\tau \geq 2$. Hence, for $\Phi^{-1}(\Phi(x))=x^{\prime}$ and $\Phi^{-1}(\Phi(y))=y^{\prime}$ we can apply the same argument above to conclude that

$$
d_{\varepsilon}\left(x^{\prime}, y^{\prime}\right) \lesssim d_{\varepsilon}(\Phi(x), \Phi(y)) .
$$


It remains to relate $d_{\varepsilon}\left(x^{\prime}, y^{\prime}\right)$ with $d_{\varepsilon}(x, y)$. As $d\left(\Phi^{-1} \circ \Phi(z), z\right) \leq \tau$ for each $z \in Y$, it follows from Lemma 3.6 that $d_{\varepsilon}\left(x^{\prime}, x\right) \lesssim e^{-\varepsilon d\left(x, y_{0}\right)}$ and $d_{\varepsilon}\left(y^{\prime}, y\right) \lesssim e^{-\varepsilon d\left(y, y_{0}\right)}$. Moreover, $d\left(x, y_{0}\right) \geq d\left(\Phi(x), x_{0}\right)-\tau$ and $d\left(y, y_{0}\right) \geq d\left(\Phi(y), x_{0}\right)-\tau$. Hence,

$d_{\varepsilon}(x, y) \leq d_{\varepsilon}\left(x, x^{\prime}\right)+d_{\varepsilon}\left(x^{\prime}, y^{\prime}\right)+d_{\varepsilon}\left(y^{\prime}, y\right) \lesssim d_{\varepsilon}(\Phi(x), \Phi(y))+e^{-\varepsilon d\left(\Phi(x), x_{0}\right)}+e^{-\varepsilon d\left(\Phi(y), x_{0}\right)}$.

Since $d(\Phi(x), \Phi(y)) \geq d(x, y)-\tau \geq 2$, we apply Lemma 3.1 to see that whenever $\beta$ is a rectifiable curve in $X$ with end points $\Phi(x)$ and $\Phi(y)$,

$$
\int_{\beta} \rho_{\varepsilon}^{X} d s \simeq \sum_{i=0}^{N-1} \rho_{\varepsilon}^{X}\left(a_{i}\right) \gtrsim \rho_{\varepsilon}^{X}(\Phi(x))+\rho_{\varepsilon}^{X}(\Phi(y)) .
$$

Taking the infimum over all such $\beta$ gives

$$
d_{\varepsilon}(\Phi(x), \Phi(y)) \gtrsim e^{-\varepsilon d\left(\Phi(x), x_{0}\right)}+e^{-\varepsilon d\left(\Phi(y), x_{0}\right)},
$$

from which we obtain the desired conclusion

$$
d_{\varepsilon}(x, y) \lesssim d_{\varepsilon}(\Phi(x), \Phi(y)) .
$$

Theorem 3.8. Let $(X, d)$ and $(Y, d)$ be two complete Gromov hyperbolic geodesic spaces, and suppose that there exists a $\tau$-rough isometry $\Phi: Y \rightarrow X$. Let $y_{0} \in Y$ and set $x_{0}=\Phi\left(y_{0}\right)$. If $\varepsilon>0$ is such that $\left(X_{\varepsilon}, d_{\varepsilon}\right)$ is a uniform domain with a uniformity constant $\lambda$, then $\left(Y_{\varepsilon}, d_{\varepsilon}\right)$ is also a uniform domain with a uniformity constant that depends solely on $\lambda$, $\varepsilon$, and $\tau$.

Proof. Let $x, y \in Y$. If $d(x, y) \leq 4+\tau$, then by Lemma 3.6 we know that the hyperbolic geodesic connecting $x$ to $y$ is a uniform curve in $\left(Y_{\varepsilon}, d_{\varepsilon}\right)$. Therefore to verify that $Y_{\varepsilon}$ is a uniform domain, it suffices to consider only points $x, y \in Y$ with $d(x, y)>4+\tau$. For such $x, y$ we have that $d(\Phi(x), \Phi(y)) \geq 4$. Let $\gamma$ be a uniform curve in $X_{\varepsilon}$ with end points $\Phi(x), \Phi(y)$. Then $\ell(\gamma) \geq 4$, and so we can apply Lemma 3.1 to $\gamma$. With $a_{i}=\gamma(i q), q=L / N$, we see that

$$
d_{\varepsilon}(x, y) \simeq d_{\varepsilon}(\Phi(x), \Phi(y)) \simeq \int_{\gamma} \rho_{\varepsilon}^{X} d s .
$$

Here we have also used Lemma 3.7. Applying Lemma 3.3 with $\Phi^{-1}: X \rightarrow Y$ playing the role of $\Phi$ there, we obtain

$$
d_{\varepsilon}(x, y) \simeq \sum_{i=0}^{N-2} \rho_{\varepsilon}^{Y}\left(\Phi^{-1}\left(a_{i}\right)\right)+\rho_{\varepsilon}^{Y}\left(\Phi^{-1}(\Phi(y))\right) .
$$

As $d\left(y, \Phi^{-1} \circ \Phi(y)\right) \leq \tau$ and $d\left(x, \Phi^{-1} \circ \Phi(x)\right) \leq \tau$, we have that

$$
d_{\varepsilon}(x, y) \simeq \rho_{\varepsilon}^{Y}(x)+\rho_{\varepsilon}^{Y}(y)+\sum_{i=1}^{N-2} \rho_{\varepsilon}^{Y}\left(\Phi^{-1}\left(a_{i}\right)\right) .
$$

Note that $d\left(a_{i}, a_{i+1}\right) \leq 2$, and therefore $d\left(\Phi^{-1}\left(a_{i}\right), \Phi^{-1}\left(a_{i+1}\right)\right) \leq 2+\tau$. Similarly, $d\left(x, \Phi^{-1}\left(a_{1}\right)\right) \leq 2+2 \tau, d\left(y, \Phi^{-1}\left(a_{N-1}\right)\right) \leq 2+2 \tau$. We set $\beta_{0}$ to be the hyperbolic geodesic with end points $\Phi^{-1}\left(a_{1}\right)$ and $x$, and set $\beta_{N-1}$ to be the hyperbolic geodesic with end points $y$ and $\Phi^{-1}\left(a_{N-1}\right)$. For $i=1, \cdots, N-2$ let $\beta_{i}$ be the hyperbolic geodesic in $Y$ with end points $\Phi^{-1}\left(a_{i}\right)$ and $\Phi^{-1}\left(a_{i+1}\right)$. By Lemma 3.6 we have that

$$
\ell_{\varepsilon}\left(\beta_{i}\right) \simeq \rho_{\varepsilon}^{Y}\left(\Phi^{-1}\left(a_{i}\right)\right) d\left(\Phi^{-1}\left(a_{i}\right), \Phi^{-1}\left(a_{i+1}\right)\right) \lesssim \rho_{\varepsilon}^{Y}\left(\Phi^{-1}\left(a_{i}\right)\right),
$$

and so

$$
d_{\varepsilon}(x, y) \gtrsim \sum_{i=0}^{N-1} \ell_{\varepsilon}\left(\beta_{i}\right)=\ell_{\varepsilon}(\beta)
$$


where $\beta$ is the concatenation of the finitely many curves $\beta_{i}, i=0, \cdots, N-1$. Thus $\beta$ is a quasiconvex curve connecting $x$ to $y$ in $Y$. We now show that this curve is a uniform curve, that is, it satisfies Condition 2 of Definition 2.4.

Let $z \in \beta$. If $z \in \beta_{0} \cup \beta_{N-1}$, then the result follows from Lemma 3.6. Thus we may assume that $z \in \beta_{i}$ for some $i \in\{1, \cdots, N-2\}$. Then by (4), Lemma 3.5, and by the uniformity of $\gamma$, we have

$$
\delta_{\varepsilon}(z) \simeq \delta_{\varepsilon}\left(\Phi^{-1}\left(a_{i}\right)\right) \simeq \delta_{\varepsilon}\left(a_{i}\right) \geq \frac{1}{\lambda} \ell_{\varepsilon}\left(\gamma\left[\Phi(x), a_{i}\right]\right) .
$$

Here we assume that $\ell_{\varepsilon}\left(\gamma\left[\Phi(x), a_{i}\right]\right)=\min \left\{\ell_{\varepsilon}\left(\gamma\left[\Phi(x), a_{i}\right]\right), \ell_{\varepsilon}\left(\gamma\left[\Phi(y), a_{i}\right]\right)\right\}$, since if this is not the case, we reverse the roles of $x$ and $y$ (and sum over all $j$ from $i$ to $N$ ) in the following estimates. A repeat of the arguments above also tell us that

$$
\ell_{\varepsilon}\left(\gamma\left[\Phi(x), a_{i}\right]\right) \simeq \sum_{j=0}^{i} \rho_{\varepsilon}^{X}\left(a_{j}\right) \simeq \sum_{j=0}^{i} \rho_{\varepsilon}^{Y}\left(\Phi^{-1}\left(a_{j}\right)\right) \gtrsim \ell_{\varepsilon}(\beta[x, z]) .
$$

Combining the above estimates, we obtain $\delta_{\varepsilon}(z) \gtrsim \ell_{\varepsilon}(\beta[x, z])$.

\section{On $(L, C)$-rough similarities}

A natural extension of the notion of rough isometries is the notion of rough similarities, which is a proper subclass of a wider class of mappings known as quasiisometries. A map $\Phi: Y \rightarrow X$ is said to be an $(L, C)$-rough similarity if $L>0$ and $C \geq 0$ are such that for every pair $x, y \in Y$ we have

$$
L d_{Y}(x, y)-C \leq d_{X}(\Phi(x), \Phi(y)) \leq L d_{Y}(x, y)+C
$$

and the Hausdorff distance between $\Phi(Y)$ and $X$ is at most $C$. Thus a natural question to ask in the setting considered in this paper is that if both $X$ and $Y$ are Gromov hyperbolic in the sense of Definition 2.1, $\varepsilon>0$ is such that $\left(X_{\varepsilon}, d_{\varepsilon}\right)$ is a uniform domain, and $\Phi: Y \rightarrow X$ is an $(L, C)$-rough similarity, then is $\left(Y_{\varepsilon}, d_{\varepsilon}\right)$ also a uniform domain? Our main theorem, Theorem 3.8, answers this in the affirmative when $L=1$. Unfortunately this theorem fails for more general $L$, as we will see in this section.

Let $Y$ be the complex unit disk, equipped with the hyperbolic metric. Then, for $R>0$, the (hyperbolic) circle $C_{R}$ centered at 0 with radius $R$ has (hyperbolic) length

$$
\ell_{Y}\left(C_{R}\right)=\frac{\pi}{2}\left(e^{2 R}-e^{-2 R}\right) .
$$

We now fix $\varepsilon>2$. Observe from [BHK] that there is some $\varepsilon_{1}>0$ such that $Y_{\varepsilon_{1}}$ is a uniform domain. Let $Z$ be the same complex unit disk, but equipped with the scaled hyperbolic metric given by $d_{Z}(z, w)=\frac{\varepsilon_{1}}{\varepsilon} d(z, w)$. We know that $Z_{\varepsilon}=Y_{\varepsilon_{1}}$, and so $Z_{\varepsilon}$ is a uniform domain. Clearly $Z$ is $\left(\frac{\varepsilon_{1}}{\varepsilon}, 0\right)$-roughly similar to $Y$. We now show that $Y_{\varepsilon}$ is not a uniform domain. For $R \gg 1$ let

$$
z_{R}=\left(\frac{e^{2 R}-1}{e^{2 R}+1}, 0\right) \quad \text { and } \quad w_{R}=\left(-\frac{e^{2 R}-1}{e^{2 R}+1}, 0\right) .
$$

The length of the hyperbolic geodesic ray $\left[z_{R},(1,0)\right) \subset \mathbf{R}$ from $z_{R}$ to $\infty$ has length $\frac{1}{\varepsilon} e^{-\varepsilon R}$, and, as $\varepsilon>2$, we have that $\partial Y_{\varepsilon}$ has only one point. Therefore,

$$
d_{\varepsilon}\left(z_{R}, w_{R}\right) \leq \frac{2}{\varepsilon} e^{-\varepsilon R} .
$$

Suppose that $Y_{\varepsilon}$ is a uniform domain with a uniformity constant $A$. Then let $\gamma$ be an $A$-uniform curve with end points $z_{R}$ and $w_{R}$. Since $\gamma$ is a compact curve, it stays within a closed hyperbolic disk centered at 0 . Let $T$ be the smallest radius of such a 
hyperbolic disk; then there is some point $z_{0}$ in $\gamma$ with $d_{Y}\left(z_{0}, 0\right)=T$. By symmetry, we may assume that $\ell_{\varepsilon}\left(\gamma_{z_{R}, z_{0}}\right) \leq \ell_{\varepsilon}\left(\gamma_{w_{R}, z_{0}}\right)$. Then

$$
\ell_{\varepsilon}\left(\gamma_{z_{R}, z_{0}}\right) \geq \int_{R}^{T} e^{-\varepsilon t} d t=\frac{e^{-\varepsilon R}-e^{-\varepsilon T}}{\varepsilon} .
$$

Thus the second condition of uniformity of $\gamma$ from Definition 2.4 implies that

$$
T \leq \frac{\log (A+1)}{\varepsilon}+R
$$

Let $\tau \geq 0$ be the largest number such that $\gamma$ lies in the complement of the hyperbolic ball centered at 0 with radius $\tau$ (with that ball being empty if $\tau=0$ ). As before, it then follows that

$$
\ell_{\varepsilon}(\gamma) \geq \frac{e^{-\varepsilon \tau}-e^{-\varepsilon R}}{\varepsilon}
$$

which, when combined together with the first condition of uniformity of $\gamma$ from Definition 2.4, tells us that

$$
\frac{e^{-\varepsilon \tau}-e^{-\varepsilon R}}{\varepsilon} \leq A d_{\varepsilon}\left(z_{R}, w_{R}\right) \leq \frac{2 A}{\varepsilon} e^{-\varepsilon R} .
$$

From this we also conclude that necessarily

$$
\tau \geq R-\frac{\log (2 A+1)}{\varepsilon}
$$

Thus the curve $\gamma$ is trapped in the annulus, centered at 0 , with (hyperbolic) inner radius $R-\frac{\log (2 A+1)}{\varepsilon}$ and (hyperbolic) outer radius $R+\frac{\log (A+1)}{\varepsilon}$. It then follows that (with $\gamma$ parametrized with respect to the arc length measured from the point of view of the hyperbolic metric)

$$
\ell_{\varepsilon}(\gamma)=\int_{\gamma} e^{-\varepsilon d_{Y}(\gamma(t), 0)} d s(t) \geq e^{-\varepsilon[R+\log (A+1) / \varepsilon]} \ell_{Y}(\gamma) \geq(A+1) e^{-\varepsilon R} \frac{\pi}{8} e^{2[R-\log (2 A+1) / \varepsilon]},
$$

where we used the fact that $R \gg 1$. Now applying the first condition of uniformity of $\gamma$ again tells us that with $C_{A}=(A+1) \frac{\pi}{8}(2 A+1)^{-2 / \varepsilon}$,

$$
C_{A} e^{-(\varepsilon-2) R} \leq A d_{\varepsilon}\left(z_{R}, w_{R}\right) \leq \frac{2 A}{\varepsilon} e^{-\varepsilon R} .
$$

As this is not possible for large $R$, it follows that $Y_{\varepsilon}$ is not uniform with uniformity constant $A$. Since the constant $A$ above is arbitrary, it follows that $Y_{\varepsilon}$ is not a uniform space.

The above computation also indicates that if $Y$ is a Gromov hyperbolic space, $C>0$ and $y_{0} \in Y$ such that for $R \gg H \gg 1$ there are points $z_{R}, w_{R} \in Y$ with $d\left(z_{R}, y_{0}\right)=d\left(w_{R}, y_{0}\right)$ so that whenever $\gamma \subset B\left(y_{0}, R+H\right) \backslash B\left(y_{0}, R-H\right)$ has $z_{R}$ and $w_{R}$ as its end points we must have $\ell(\gamma) \geq c_{H} e^{C R}$, then $Y_{\varepsilon}$ cannot be a uniform space when $\varepsilon>C$. Should $Y$ be a smooth hyperbolic manifold, the smallest choice of such $C$ is associated with the curvature of $Y$; however, as we are merely concerned with the large scale behavior here, this association is not straight-forward. In the setting of (non-smooth) metric spaces a similar limitation on $\varepsilon$ in terms of some notion of curvature might be possible. Indeed, from the work of [BHK] we know that for each $\delta>0$ there is some $\varepsilon_{0}(\delta)>0$ such that if $Y$ is Gromov hyperbolic with hyperbolicity constant $\delta$ as in Definition 2.1, then $Y_{\varepsilon}$ is a uniform domain whenever $\varepsilon \leq \varepsilon_{0}(\delta)$. The estimates for $\varepsilon_{0}(\delta)$ are not explicit in [BHK], and may perhaps not even be optimal. However, the results of [BHK] indicate a link between some notion of curvature of $Y$ and the supremum of all $\varepsilon>0$ for which $Y_{\varepsilon}$ is a uniform domain. As this link is not 
well understood so far, and the estimates for the limitation $\varepsilon_{0}(\delta)$ in $[\mathrm{BHK}]$ are not known to be optimal, Theorem 3.8 gives a way of verifying uniformity of $Y_{\varepsilon}$ without linking it to $\delta$ but instead comparing it to all other locally compact geodesic metric spaces that are roughly isometric to $Y$.

\section{References}

[A1] AiKaWA, H.: Equivalence between the boundary Harnack principle and the Carleson estimate. - Math. Scand. 103:1, 2008, 61-76.

[A2] Aikawa, H.: Potential-theoretic characterizations of nonsmooth domains. - Bull. London Math. Soc. 36:4, 2004, 469-482.

[BB] BJÖRN, A., and J. BJÖRN: Nonlinear potential theory on metric spaces. - EMS Tracts Math. 17, Eur. Math. Soc., Zürich, 2011.

[BBS] BJörn, A., J. BJöRn, and N. Shanmugalingam: Extension and trace results for doubling metric measure spaces and their hyperbolic fillings. - Preprint, https://arxiv.org/ abs $/ 2008.00588$.

[BBS2] Buörn, A., J. Buörn, and N. Shanmugalingam: Bounded geometry and $p$-harmonic functions under uniformization and hyperbolization. - J. Geom. Anal. (to appear), doi: https://doi.org/10.1007/s12220-020-00477-0.

[BSh] BJörn, J., and N. Shanmugalingam: Poincaré inequalities, uniform domains and extension properties for Newton-Sobolev functions in metric spaces. - J. Math. Anal. Appl. 332:1, 2007, 190-208.

[Bo] Bonk, M.: Quasi-geodesic segments and Gromov hyperbolicity. - Geom. Dedicata 62:3, 1996, 281-298.

[BHK] Bonk, M., J. Heinonen, and P. Koskela: Uniformizing Gromov hyperbolic spaces. Astérisque 270, 2001.

[BKR] Bonk, M., P. Koskela, and S. Rohde: Conformal metrics on the unit ball in Euclidean space. - Proc. London Math. Soc. (3) 77:3, 1998, 635-664.

[BS] Bonk, M., and O. Schramm: Embeddings of Gromov hyperbolic spaces. - Geom. Funct. Anal. 10:2, 2000, 266-306.

[BP] Bourdon, M., and H. PAjot: Cohomologie $l_{p}$ et espaces de Besov. - J. Reine Angew. Math. 558, 2003, 85-108.

[Bow] Bowditch, B.: A course on geometric group theory. - MSJ Mem., Mathematical Society of Japan, 2006.

[BH] Bridson, M.R., and A. HaEfliger: Metric spaces of non-positive curvature. Grundlehren Math. Wiss. 319, Springer-Verlag, Berlin, 1999.

[BuS] Buyalo, S., and V. Schroeder: Elements of asymptotic geometry. - EMS Monogr. Math., Eur. Math. Soc., Zürich, 2007.

[CDP] Coornaert, M., T. Delzant, and A. Papadopoulos: Géométrie et théorie des groupes. Les groupes hyperboliques de Gromov. - Lecture Notes in Math. 1441, Springer-Verlag, Berlin, 1990.

[GeO] Gehring, F. W., and B. G. OsGood: Uniform domains and the quasihyperbolic metric. - J. Anal. Math. 36, 1979, 50-74.

[GH] Ghys, E., and P. DE la Harpe: Le bord d'un espace hyperbolique. - In: Sur les groupes hyperboliques d'aprés Mikhael Gromov (Bern, 1988), Progr. Math. 83, 1990, 117-134.

[GKS] Gogatishvili, A., P. Koskela, N. Shanmugalingam: Interpolation properties of Besov spaces defined on metric spaces. - Math. Nachr. 283, 2010, 215-231.

[Gr] Gromov, M.: Hyperbolic groups. - In: Essays in group theory, Math. Sci. Res. Inst. Publ. $8,1987,75-263$. 
[H] Herron, D.: Conformal deformations of uniform Loewner spaces. - Math. Proc. Cambridge Philos. Soc. 136:2, 2004, 325-360.

[HK] HajŁasz, P., and P. Koskela: Sobolev met Poincaré. - Mem. Amer. Math. Soc. 145:688, 2000 .

[KP] Kenig, C., and J. Pipher: The $h$-path distribution of the lifetime of conditioned Brownian motion for nonsmooth domains. - Probab. Theory Related Fields 82:4, 1989, 615-623.

[KT] Kenig, C., and T. Toro: Free boundary regularity for harmonic measures and Poisson kernels. - Ann. of Math. (2) 150:2, 1999, 369-454.

[KL] Koskela, P., and P. LAmmi: Gehring-Hayman theorem for conformal deformations. Comment. Math. Helv. 88:1, 2013, 185-203.

[LlMS] Lewis, J., P. Lindqvist, J. Manfredi, and S. Salsa. Regularity estimates for nonlinear elliptic and parabolic problems. - In: Notes of the CIME course held in Cetraro (2009), edited by U. Gianazza and J. Lewis, Lecture Notes in Math. 2045, Fondazione CIME/CIME Foundation Subseries, Springer, Heidelberg, Centro Internazionale Matematico Estivo (C.I.M.E.), Florence, 2012.

[Mar] Martio, O.: Definitions for uniform domains. - Ann. Acad. Sci. Fenn. Ser. A I Math. 5:1, 1980, 197-205.

[MS] Martio,O., and J. Sarvas: Injectivity theorems in plane and space. - Ann. Acad. Sci. Fenn. Ser. A I Math. 4:2, 1979, 383-401.

Received 20 December 2019 • Accepted 25 May 2020 